\title{
Identification of a Novel Microtubule-Binding Protein in Giardia lamblia
}

\author{
Juri Kim, Soon-Jung Park* \\ Department of Environmental Medical Biology and Institute of Tropical Medicine, Brain Korea 21 PLUS Project for Medical Science, Yonsei University \\ College of Medicine, Seoul 03722, Korea
}

\begin{abstract}
Giardia lamblia is a protozoan that causes diarrheal diseases in humans. Cytoskeletal structures of Giardia trophozoites must be finely reorganized during cell division. To identify Giardia proteins which interact with microtubules (MTs), Giardia lysates were incubated with in vitro-polymerized MTs and then precipitated by ultracentifugation. A hypothetical protein (GL50803_8405) was identified in the precipitated fraction with polymerized MTs and was named GIMBP1 (G. lamblia microtubule-binding protein 1). Interaction of GIMBP1 with MTs was confirmed by MT binding assays using recombinant GIMBP1 (rGIMBP1). In vivo expression of GIMBP1 was shown by a real-time PCR and western blot analysis using anti-rGIMBP1 antibodies. Transgenic G. lamblia trophozoites were constructed by integrating a chimeric gene encoding hemagglutinin (HA)-tagged GIMBP1 into a Giardia chromosome. Immunofluorescence assays of this transgenic G. lamblia, using anti-HA antibodies, revealed that GIMBP1 mainly localized at the basal bodies, axonemes, and median bodies of G. lamblia trophozoites. This result indicates that GIMBP1 is a component of the G. lamblia cytoskeleton.
\end{abstract}

Key words: Giardia lamblia, microtubule-binding protein 1

\section{INTRODUCTION}

Giardia lamblia infects humans as cysts that are converted into trophozoites, which multiply by binary fission in human intestines and cause diarrheal disease. A trophozoite of G. lamblia has 2 nuclei and characteristic cytoskeletal structures such as a ventral disc, a median body, 4 pairs of flagella, and a funis [1]. Positioning of these structures in the dividing Giardia cells must be finely coordinated for successful proliferation. In eukaryotic organisms, microtubules (MTs) play an essential role in the coordinated movement of cellular structures by maintaining equilibrium between polymerization and depolymerization [2]. Growing and shortening of MTs is mediated by MT-associated proteins, including end-binding 1 (EB1), which is a plus-end tracking protein [3].

An EB1 homologous protein (GlEB1) was found in the flagellar tips, median bodies, and mitotic spindles of G. lamblia $[4,5]$. The role of GlEB1 was assessed by complementation assays using a BIM1 mutant of Saccharomyces cerevisiae, in which

- Received 3 December 2015, revised 28 April 2016, accepted 26 May 2016.

*Corresponding author (sjpark615@yuhs.ac)

(c) 2016, Korean Society for Parasitology and Tropical Medicine

This is an Open Access article distributed under the terms of the Creative Commons Attribution Non-Commercial License (http://creativecommons.org/licenses/by-nc/4.0) which permits unrestricted non-commercial use, distribution, and reproduction in any

medium, provided the original work is properly cited. proper positioning of the nucleus was abolished [6]. Biochemical characterization of GIEB1 was performed to define the domains responsible for MT binding and dimerization [5].

Investigations on the Giardia cytoskeleton have focused on its unique structures such as the ventral disc and median body. Tubulin and Giardia-specific giardin proteins were identified, via Triton X-100 extraction, as the main components of $G$. lamblia ventral disc [7]. Recent technical progress in proteomic analysis has led to the discovery of additional proteins associated with the ventral disc, whose function is yet to be defined [8]. In addition, shotgun proteomics along with GFP-tagging of the purified ventral disc of G. lamblia facilitated the identification of 18 novel disc-associated proteins [9]. One of these disc-associated proteins, DAP116343, was also found in the median body and knockdown of this protein by morpholinos resulted in aberrant disc formation in G. lamblia [10].

Thus, dynamic MTs are expected to mediate cell division in G. lamblia. Various MT-binding proteins may play roles in this process. In this study, a novel MT-binding protein was discovered in Giardia lysates, using in vitro-polymerized MTs.

\section{MATERIALS AND METHODS}

Giardia cell culture and preparation of Giardia extracts

Trophozoites of the G. lamblia WB strain (ATCC30957; 
American Type Culture Collection, Manassas, Virginia, USA) were grown for $72 \mathrm{hr}$ in TYI-S-33 medium ( $2 \%$ casein digest, $1 \%$ yeast extract, $1 \%$ glucose, $0.2 \% \mathrm{NaCl}, 0.2 \%$ L-cysteine, $0.02 \%$ ascorbic acid, $0.2 \% \mathrm{~K}_{2} \mathrm{HPO}_{4}, 0.06 \% \mathrm{KH}_{2} \mathrm{PO}_{4}, 10 \%$ calf serum, and $0.5 \mathrm{mg} / \mathrm{ml}$ bovine bile, $\mathrm{pH}$ 7.1) [11]. They were then resuspended in PBS ( $137 \mathrm{mM} \mathrm{NaCl}, 2.7 \mathrm{mM} \mathrm{KCl}, 10.1 \mathrm{mM} \mathrm{Na} \mathrm{H}_{-}$ $\mathrm{PO}_{4}$, and $\left.2 \mathrm{mM} \mathrm{KH} 2 \mathrm{PO} 4, \mathrm{pH} 7.4\right)$, and lysed by sonication.

\section{MT-binding assay}

The binding of Giardia lysates to polymerized MTs was performed in vitro using the Microtubule-Binding Protein SpinDown Assay Kit BK029 (Cytoskeleton, Denver, Colorado, USA). MTs were assembled from $100 \mu \mathrm{g}$ of pure tubulin (isolated from bovine brain; Cytoskeleton) in $20 \mu \mathrm{l}$ of PEM [80 $\mathrm{mM}$ piperazine-N,N'-bis(2-ethanesulfonic acid), pH 6.8, 1 mM EGTA, and $1 \mathrm{mM} \mathrm{MgCl}_{2}$ in the presence of $1 \mathrm{mM}$ GTP and $5 \%$ glycerol at $35^{\circ} \mathrm{C}$ for $20 \mathrm{~min}$, and immediately stabilized in $200 \mu \mathrm{l}$ of warm PEM-20 $\mu \mathrm{M}$ taxol (Cytoskeleton). Twenty $\mu$ moles of the MTs were incubated with $100 \mu \mathrm{g}$ of Giardia lysate in a total volume of $50 \mu \mathrm{l}$ at $25^{\circ} \mathrm{C}$ for $40 \mathrm{~min}$. The reaction mixtures were then centrifuged with a $50 \%$ glycerol cushion-PEM-taxol mixture, at $100,000 \mathrm{~g}$ at $25^{\circ} \mathrm{C}$ for $40 \mathrm{~min}$ in an ultracentrifuge (Hitachi Koki, Tokyo, Japan). The resulting pellet fraction was then resolved on an $8 \%$ polyacrylamide gel and visualized by silver staining. The same amount of Giardia extract was precipitated by ultracentrifugation, and compared side-by-side with the extracts precipitated with MTs.

\section{Liquid chromatography mass spectrometry}

The protein band present in the MT fraction was excised and digested with trypsin. The trypsin-treated proteins were analyzed by quadrupole time-of-flight (Q-TOF) mass spectrometry (MS) in addition to matrix-assisted laser desorption ionizationTOF MS (MALDI-TOF MS). Product ion spectra were collected in the information-dependent acquisition mode and were analyzed with an Agilent 6530 accurate-mass Q-TOF MS. For the Q-TOF liquid chromatography-tandem MS (LC-MS/MS) data sets, tandem mass spectra were submitted to our MASCOT inhouse database search engine (NCBI NR database downloaded on 31 July 2009). For protein identification, a MASCOT ion score of $>37$ was used as the criterion for a meaningful result.

\section{Expression and purification of recombinant GIMBP1 (rGIMBP1)}

A 1,338 bp DNA fragment encoding the GlMBP1 open reading frame (ORF) was amplified by PCR from the genomic DNA of G. lamblia, using 2 primers, 8405F and 8405R (Table 1), and cloned into pET21b (Novagen, Darmatadt, Germany),

Table 1. Strains, primers, and plasmids used in this study

\begin{tabular}{|c|c|c|}
\hline Strains/primers/ plasmids & Relevant characteristics ${ }^{a}$ & Source or reference \\
\hline \multicolumn{3}{|l|}{ G. lamblia } \\
\hline ATCC 30957 & Clinical isolate & ATCC \\
\hline $\mathrm{DH} 5 \mathrm{a}$ & supE44 DlacU169 (F80 lacZ DM15) hsdR17 recA1 endA1 gyrA96 thi-1 relA1 & Invitrogen \\
\hline BL21 (DE3) & $F^{\prime}$, ompt, hsdSB(rB-mB-) gal, dcm (DE3) & Invitrogen \\
\hline \multicolumn{3}{|l|}{ Primers } \\
\hline $8405 F$ & GCGAATTCGATGTCGATGGACGTTCCTA (ECORI) & \\
\hline $8405 R$ & AGTाAGCGGCCGCGCGCTाGAGCCACACTCCA (Notl) & \\
\hline GIMBP-F & CGATCCATGGCCGGCTCTTGTCCAACTGCT (Ncol) & \\
\hline \multirow[t]{3}{*}{ GIMBP-HA-R } & GTTACGCGGCCGCTTAAGCGTAATCTGGAACATCG & \\
\hline & TATGGGTAAGCGTAATCTGGAACATCGTATGGGTAA & \\
\hline & GCGTAATCTGGAACATCGTATGGGTAGCGCTाTGAGCCACACTCCA (Ncol) & \\
\hline pac down & CGCGAATTCTCAGGCACCGGGCTT & \\
\hline RT-tim_R & CTATGTACGGGTCTTCGTAAGA & \\
\hline RT-GIMBP-F & GATGAAGTAGATAAGGCGGCA & \\
\hline RT-GIMBP-R & GAGCCACACTCCATACAGAAT & \\
\hline \multicolumn{3}{|l|}{ Plasmids } \\
\hline pET21b & Expression vector for a histidine-tagged protein & Novagen \\
\hline pETGIMBP1 & pET21b, 1,338 bp encoding GIBMP1 (GiardiaDB; GL50803_8405) & This study \\
\hline pGFP.pac & Shuttle vector, $A m p^{R}$, pac gene & Singer et al. [13] \\
\hline pGIMBP1HA.pac & pGFP.pac, 1,538 bp encoding GIMBP1 from its own promoter & This study \\
\hline
\end{tabular}

anderlined bases indicate a restriction enzyme site. 
resulting in pETGIMBP1. The rGIMBP1 was expressed in Escherichia coli BL21 (DE3), with $0.5 \mathrm{mM}$ ITPG at $30^{\circ} \mathrm{C}$ for $3 \mathrm{hr}$, and purified using TALON Metal affinity chromatography, as described by the manufacturer (Clontech, Mountain View, California, USA).

\section{Quantitative measurement of GIMBP1 transcript}

The level of GIMBP1 mRNA expression was evaluated by realtime PCR. Total RNA was isolated from G. lamblia, using TRIzol (Invitrogen, Carlsbad, California, USA). cDNA was synthesized from $5 \mu \mathrm{g}$ of RNA using the ImProm-II ${ }^{\mathrm{TM}}$ RT system (Promega, Madison, Wisconsin, USA) following the manufacturer's directions. cDNA was then analyzed in the Light Cycler 480 II RealTime PCR System (Roche Applied Science, Mannheim, Germany) using LightCycler 490 DNA SYBR Green I Master (Roche Applied Science). Conditions for real-time PCR were as follows: pre-incubation at $95^{\circ} \mathrm{C}$ for 5 min followed by 45 amplification cycles of $95^{\circ} \mathrm{C}$ for $10 \mathrm{sec}, 56^{\circ} \mathrm{C}$ for $20 \mathrm{sec}$, and $72^{\circ} \mathrm{C}$ for $10 \mathrm{sec}$. Real-time PCR was carried out in triplicate in a 96-well plate using the specific primers listed in Table 1. The tim gene encoding triose-1-phosphate isomerase of G. lamblia was used as an endogenous control for the reactions.

\section{Formation of anti-rGIMBP1 antibodies}

Histidine-tagged rGlMBP1 was expressed in E. coli BL21 (DE3) with the addition of $0.5 \mathrm{mM}$ IPTG at $37^{\circ} \mathrm{C}$ for $3 \mathrm{hr}$. The rGIMBP1 protein was excised from the acrylamide gel, and used to immunize Sprague-Dawley rats (2-week-old, female) to produce polyclonal antibodies as described previously [12].

\section{Western blot analysis}

Ten $\mu$ g of purified rGIMBP1 reacted with $20 \mu \mathrm{M}$ polymerized MTs was used for MT-binding assays. After ultracentrifugation, the resulting soluble and pellet fractions were separated on $12 \%$ acrylamide gel, and then transferred to nitrocellulose membranes (Milipore, Bedford, Massachusetts, USA). The membrane was incubated with anti-histidine antibodies (1:5,000 dilution; IG Therapy, Chunchon, Korea) in a blocking solution (Tris-buffered saline containing 5\% skim milk, and 0.1\% Tween 20) at $4^{\circ} \mathrm{C}$ overnight. Following incubation with alkaline phosphatase-conjugated anti-mouse IgG (1:2,000 dilution; Sigma, St. Louis, Missouri, USA), the immunoreactive protein was visualized in a nitro blue tetrazolium/5'-bromo-4-chloro-3-indolyphosphate system (Promega).

\section{Integration of HA-tagged glmbp1 into a Giardia chromosome}

To tag a hemagglutinin (HA) epitope to the C-terminal portion of the glmbp1 gene, a 1,538 bp DNA fragment containing the promoter (200 bp) and ORF of the glmbp1 gene was amplified from G. lamblia WB genomic DNA by PCR, using 2 primers, GIMBP-F and GIMBP-HA-R (Table 1). NcoI and NotI sites, located at the ends of the resulting glmbp1 DNA, were used for cloning into the corresponding sites of plasmid pGFP. pac [13], resulting in the plasmid pGlMBP1HA.pac. This construct was verified by DNA sequencing (Macrogen, Seoul, Korea). The plasmid was linearized using its unique PstI site, and $10 \mu \mathrm{g}$ of linear DNA was then introduced into Giardia trophozoites. Briefly, the trophozoites were grown for $72 \mathrm{hr}$ in normal TYI-S-33 medium. Transfection of linearized DNA into G. lamblia trophozoites was performed by electroporation under the following conditions: 350 volts, $1000 \mu \mathrm{F}$, and $700 \Omega$ (BioRad, Hercules, California, USA). Trophozoites harboring pGlMBP1HA.pac were selected by adding puromycin (AG Scientific, San Diego, California, USA) to the TYI-S-33 medium at a final concentration of $10 \mu \mathrm{g} / \mathrm{ml}$. After 7 or 8 days of cultivation, resistant cells were recovered. To confirm that the construct was integrated into the Giardia genome in the transfected cells, PCR was performed for the isolated genomic DNA from pGIMBP1HA. pac-transfected cells, using a common 5'-primer (GIMBP-F) and two 3'-primers (GIMBP-HA-R or pac down) (Table 1). PCR conditions were as follows: pre-denaturation at $94^{\circ} \mathrm{C}$ for $5 \mathrm{~min}$ followed by 35 cycles of $94^{\circ} \mathrm{C}$ for $1 \mathrm{~min}, 56^{\circ} \mathrm{C}$ for $1 \mathrm{~min}$, and $72^{\circ} \mathrm{C}$ for $1 \mathrm{~min} 30 \mathrm{sec}$, and post-elongation at $72^{\circ} \mathrm{C}$ for 10 min using a Veriti Thermal Cycler (Life Technologies, Foster City, California, USA).

To determine whether HA-tagged GlMBP1 was expressed in the transfected Giardia, cell extracts were prepared from $G$. lamblia WB and puromycin-resistant G. lamblia, and then were analyzed by sodium dodecyl-polyacrylamide gel electrophoresis (SDS-PAGE). The resulting membrane was incubated with rat anti-GlMBP1 antibodies (1:1,000), followed by incubation with horseradish peroxidase-conjugated anti-rat-IgG antibodies $(1: 1,000)$. Immunoreactive proteins were visualized using an Enhanced Chemiluminescence System (Youngin Frontier Company, Seoul, Korea). The membrane was incubated in a stripping buffer (ATTO Corporation, Tokyo, Japan) at room temperature for $30 \mathrm{~min}$, and reacted with polyclonal G. lamblia anti-a-tubulin antibodies $(1: 10,000)$ as a loading control. 
Immunofluorescence assay (IFA)

To examine the localization of GIMBP1 in G. lamblia expressing HA-tagged GIMBP1, the cells were attached to glass slides coated with L-lysine in a humidified chamber. The attached cells were fixed with chilled $100 \%$ methanol at $-20^{\circ} \mathrm{C}$ for 10 min and then permeabilized with PBS/0.5\% Triton X-100 for $10 \mathrm{~min}$. After 1-hr incubation in blocking buffer [PBS, 5\% goat serum, and 3\% bovine serum albumin (BSA)], the cells were reacted overnight with mouse anti-HA antibodies (1:50; Sigma). Following 3 times 5-min washes with PBS, the cells were incubated with AlexaFluor 488-conjugated anti-mouse IgG (1:200; Molecular Probes, Grand Island, New York, USA) at $37^{\circ} \mathrm{C}$ for $1 \mathrm{hr}$. Cells were mounted on slides with VECTASHIELD Anti-fade Mounting Medium with 4',6-diamidino-2phenylindole (Vector Laboratories, Burlingame, California, USA) and then were observed with an Axiovert 200 fluorescence microscope (Carl Zeiss, Oberkochen, Germany).

\section{RESULTS}

\section{Isolation of a G. lamblia MT-binding protein 1, GIMBP1}

The pattern seen from proteins precipitated with MTs was almost identical to that of the control, except for 2 protein bands (Fig. 1A). The larger and more abundant of these proteins was $55 \mathrm{kDa}$ in size and was believed to be the tubulin monomer used in the MT-binding assay, while the smaller and less abundant protein was thought to be an MT-binding protein derived from Giardia extracts. An extended view of the separated proteins between $55 \mathrm{kDa}$ and $25 \mathrm{kDa}$ revealed a protein of $<40 \mathrm{kDa}$ that was among the proteins precipitated with MTs, but was absent in the control proteins as indicated with an arrow (Fig. 1B).

This protein was excised and processed for Q-TOF LC MS/ MS analysis (Fig. 1C). It was identified as a hypothetical protein, annotated as GL50803_8405, and named G. lamblia MTbinding protein 1, GIMBP1. The putative protein had a molec-

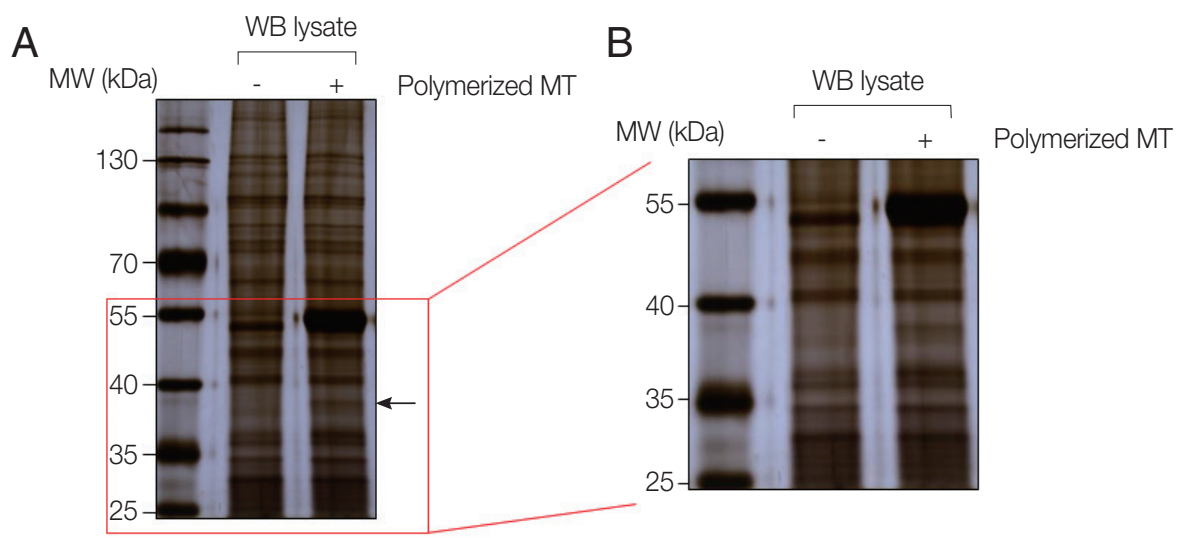

\begin{tabular}{lccccc}
\hline Protein name & MW (kDa) & pl $^{\mathrm{a}}$ & Swquence Coverage $^{\mathrm{a}}$ & Matcged peptide number $^{\mathrm{a}}$ & ${\text { Score }\left(\text { valid value }^{\mathrm{b}}\right.}^{\mathrm{a}}$ \\
$\begin{array}{l}\text { Hypothetucal protein } \\
\text { GL50803_8405 }\end{array}$ & 48 & 9.65 & $16 \%$ & 6 & $66(37)$ \\
\hline
\end{tabular}

aMatching masses from liquid chromatography-mass spectrometry when searching against all entries in NCBi using Mascot search engine. brobability value value obtained from Mascot search.

Fig. 1. Identification of GIMBP1, an MT-binding protein from Giardia extracts via in vitro MT-binding assays. (A) Trophozoites of G. lamblia WB were resuspended in PBS, and lysed by sonication. The binding of Giardia lysates to polymerized MTs was assessed in vitro using a Microtubule-Binding Protein Spin-Down Assay Kit BK029 (Cytoskeleton). Twenty umoles of MTs was incubated with $100 \mu \mathrm{g}$ of Giardia lysates in a total volume of $50 \mu \mathrm{l}$ at room temperature for $40 \mathrm{~min}$. The reaction mixtures were then centrifuged through a $50 \%$ glycerol cushion-PEM-taxol mixture at $100,000 \mathrm{~g}$ at $25^{\circ} \mathrm{C}$ for $40 \mathrm{~min}$ using an ultracentrifuge. The pellet fraction was then resolved on $8 \%$ SDSPAGE and visualized by silver staining. A sample with the same amount of Giardia extracts was precipitated by ultracentrifugation and compared side-by-side with the extracts precipitated with MTs. (B) An extended view of the SDS-PAGE gel showing proteins between $55 \mathrm{kDa}$ and $25 \mathrm{kDa}$. (C) The protein band present only in the MT fraction was excised and digested with trypsin. The trypsin-treated proteins were analyzed by quadrupole time-of-flight (Q-TOF), in addition to matrix-assisted laser desorption ionization-TOF mass spectrometry (MALDI-TOF MS). For the Q-TOF liquid chromatography-tandem MS (LC-MS/MS) data sets, tandem mass spectra were submitted to our MASCOT in-house database search engine (NCBI NR database downloaded on 31 July 2009). For protein identification, a MASCOT ion score of $>37$ was used as the criterion for a meaningful result. 
ular weight of $48 \mathrm{kDa}$ and a pI value of 9.65. The Q-TOF LCMS/MS analysis indicated 6 matched peptides, 16\% coverage, and a meaningful MASCOT score of 66 . Interestingly, the putative size of this annotated protein was $48 \mathrm{kDa}$ whereas the excised protein was smaller $(<40 \mathrm{kDa})$. This result suggested the possibility that the excised protein was a degradation product of GIMBP1.

The open reading frame (ORF) encoding GIMBP1 was made of 445 amino acids. A BlastP search of the protein database of other metazoan organisms yielded only homologous protein except a homologous protein (SS50377_11087) in Spironucleus salmonicida, a protozoan pathogen in salmons. A domain search of GlMBP1, as well as the homologous protein in S. salmonicida, did not show any MT-binding domains such as calponin homology, cytoskeletal associated protein-glycine rich, and tumor overexpressed gene domains, which are con-

\section{A}

Coomassie staining

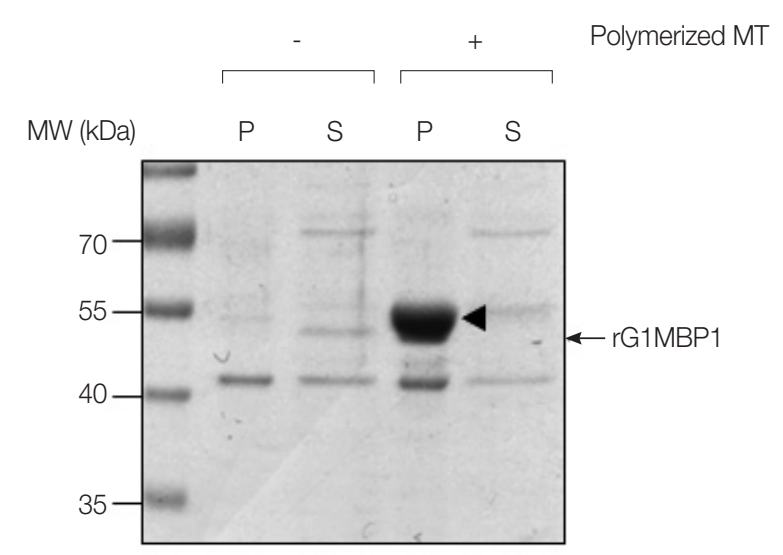

B

Anti-histidine

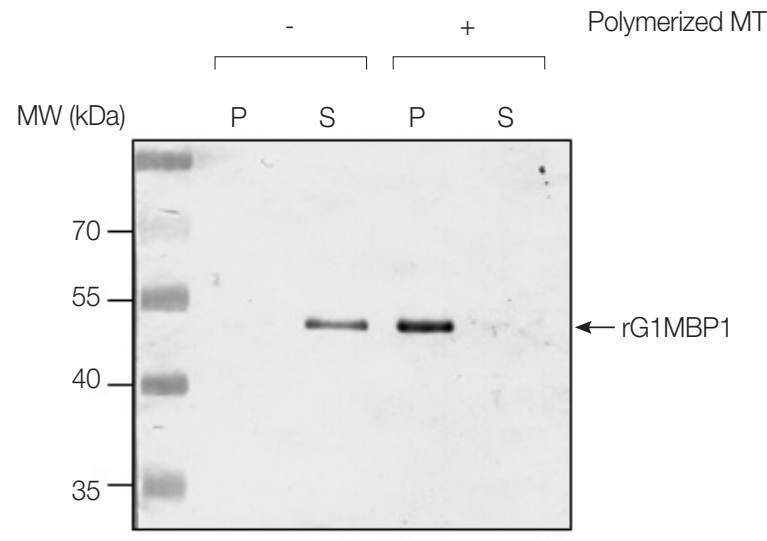

Fig. 2. In vitro MT-binding assays using rGIMBP1. Ten $\mu$ g of rGIMBP1 was incubated without or with taxol-stabilized bovine MTs (20 $\mu \mathrm{M})$, divided into pellet $(\mathrm{P})$ and soluble $(\mathrm{S})$ fractions by ultracentrifugation, and then separated by $12 \%$ SDS-PAGE. (A) A SDS-PAGE gel stained with Coomassie brilliant blue. (B) Western blot using anti-histidine antibodies (1:5,000 dilution). An arrowhead (about 55 kDa) indicates MTs, whereas arrows denote rGIMTBP.

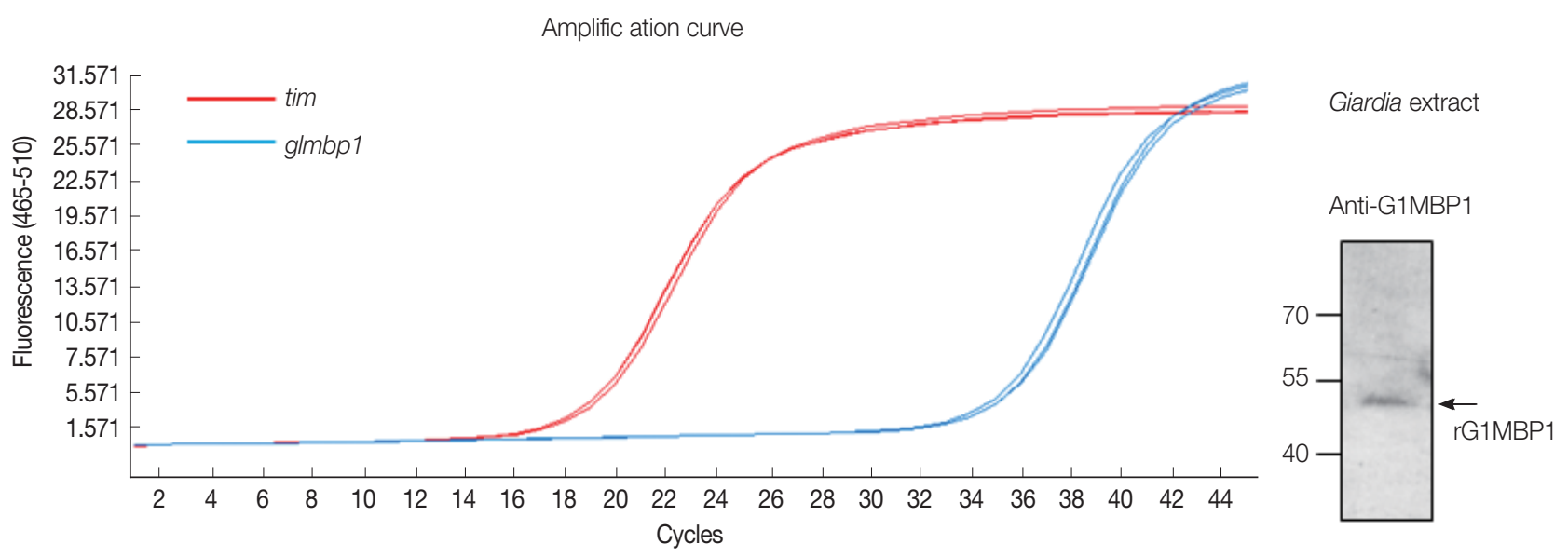

Fig. 3. In vivo expression of GIMBP1 in G. lamblia trophozoites. (A) Quantitative measurement of GIMBP1 transcripts. Total RNA was isolated from $\mathrm{G}$. lamblia using TRIzol. cDNA was synthesized from $5 \mu \mathrm{g}$ of RNA using the ImProm- $\|^{\mathrm{TM}}$ RT system and then analyzed with the Light Cycler 480 II Real-Time PCR System using LightCycler 490 DNA SYBR Green I Master (Roche Applied Science). Conditions for real-time PCR were as follows: pre-incubation at $95^{\circ} \mathrm{C}$ for 5 min followed by 45 amplification cycles of $95^{\circ} \mathrm{C}$ for $10 \mathrm{sec}, 56^{\circ} \mathrm{C}$ for 20 $\mathrm{sec}$, and $72^{\circ} \mathrm{C}$ for $10 \mathrm{sec}$. Real-time PCR was carried out in triplicate in a 96-well plate using the specific primers listed in Table 1. The tim gene encoding triose-1-phosphate isomerase of $\mathrm{G}$. lamblia was used as an endogenous control for the reactions. (B) Western blot analysis. Ten $\mu \mathrm{g}$ of Giardia extracts was separated by $12 \%$ SDS-PAGE and transferred onto a nitrocellulose membrane. The membrane was incubated with anti-GIMBP1 antibodies (1:1,000 dilution), followed by secondary antibodies (1:1,000 dilution). 


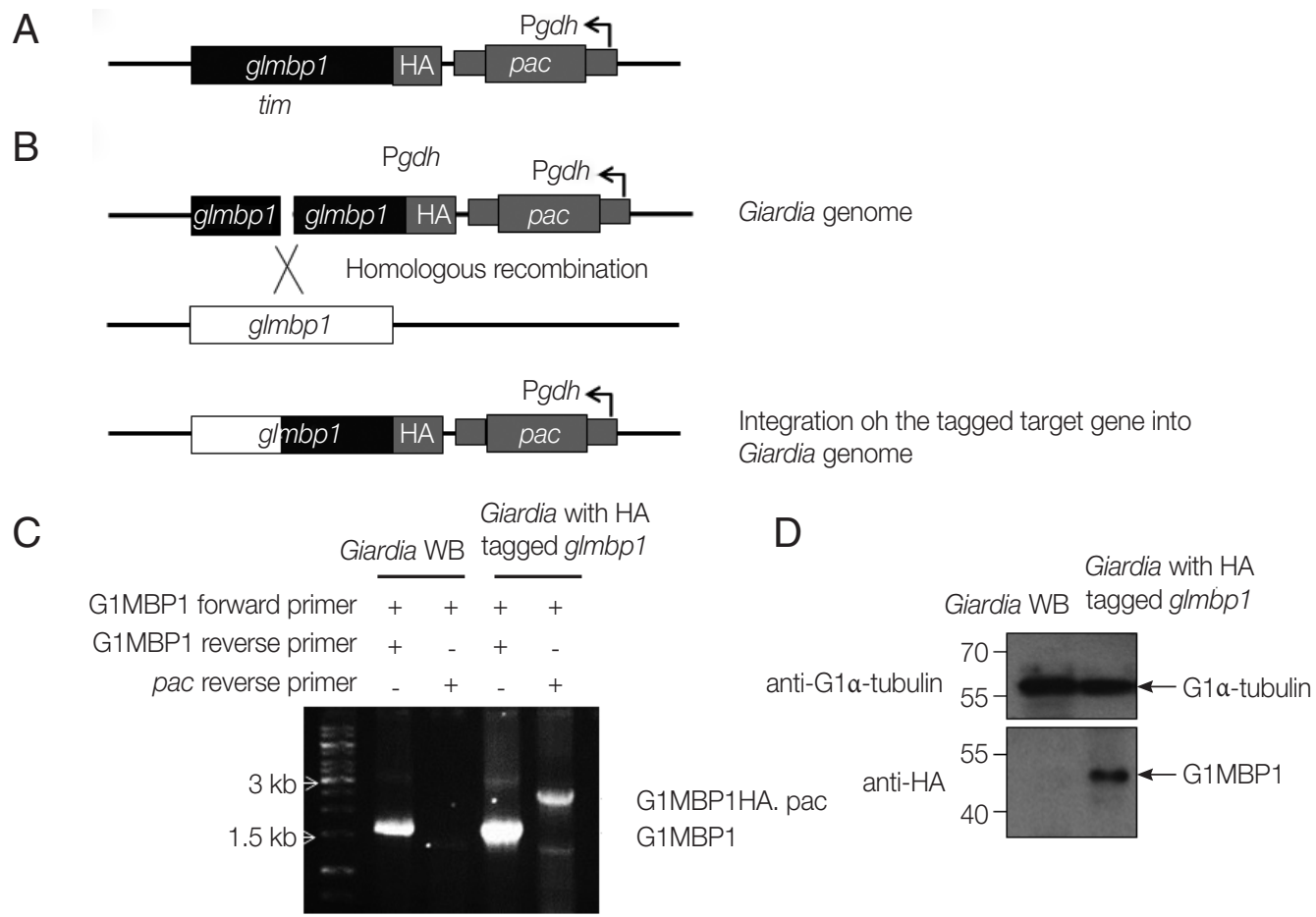

Fig. 4. Expression of HA-tagged GIMBP1 in G. lamblia. (A) A schematic diagram of the plasmid pGIMBP1HA.pac. This plasmid contains the in-frame $\mathrm{N}$-terminal end of the glmbp 1 gene with $3 \mathrm{HA}$ epitopes and the puromycin N-acetyltransferase gene (pac) cassette as a selective marker expressed from the glutamate dehydrogenase gene (gdh) promoter, Pgdh. (B) A strategy to integrate pGIMBP1HA.pac into a Giardia chromosome. Closed boxes show the plasmid sequences, and open boxes indicate Giardia chromosomal sequences. HA-tagging sequences and the pac cassette are indicated by gray boxes. (C) Genomic PCR analysis. PCR was performed using the GIMBP-F and GIMBP-HA-R or pac down primers on genomic DNA from G. lamblia WB cells or pGIMBP1HA.pac-transfected cells. (D) Western blot analysis. Ten $\mu \mathrm{g}$ of Giardia extracts (G. lamblia WB cells or pGIMBP1HA.pac-transfected cells) was separated by $12 \%$ SDS-PAGE and transferred to nitrocellulose membranes. The membrane was incubated with anti-HA antibodies (1:1,000 dilution), and then secondary antibodies (1:1,000 dilution).

served in mammalian and yeasts. The only domains found in these proteins were C2HC-type zinc-finger domains.

\section{Confirmation of the MT-binding ability of GIMBP1}

To confirm the association between GIMBP1 and MTs, an MT-binding assay was performed with recombinant GIMBP1 (rGlMBP1) and in vitro polymerized MTs. In the absence of MTs, histidine-tagged rGlMBP1 was found in the soluble fraction by Coomassie-staining and western blot analysis using anti-histidine antibodies (Fig. 2A, B, respectively). Upon incubation with polymerized MTs, rGIMBP1 was found in the pellet fraction along with tubulin monomer (Fig. 2A). Because the sizes of tubulin and rGIMBP1 were similar, it was difficult to distinguish one from the other in the SDS-PAGE gel. However, western blotting using anti-histidine antibodies clearly showed that rGIMBP1 precipitated with polymerized MTs, indicating that rGIMBP1 binds to MTs (Fig. 2B).

\section{In vivo expression of GIMBP1 in G. lamblia}

Even though GIMBP1 was identified as an MT-binding protein in G. lamblia extracts, whether this protein is expressed in vivo in G. lamblia still needed to be determined. To assess the expression of $g l m b p 1$ in Giardia, the level of $g l m b p 1$ transcripts was monitored by real-time reverse transcriptase PCR of trophozoites using primers specific to the glmbp1 gene (Fig. 3A). The level of tim transcript encoding triose 1-phosphate isomerase was used to normalize other transcripts from identical RNA samples [14]. The crossing point-PCR cycle (Cp) values were estimated as 18.8 and 35.6 for tim and glmbp1, respectively. Thus, the level of $g l m b p 1$ transcript expression was lower than that of tim, but glmbp1 transcripts were present in G. lamblia trophozoites.

In addition, the presence of GIMBP1 in Giardia trophozoites was demonstrated by western blot analysis of Giardia extracts with anti-GlMBP1 antibodies. An immunoreactive protein of 

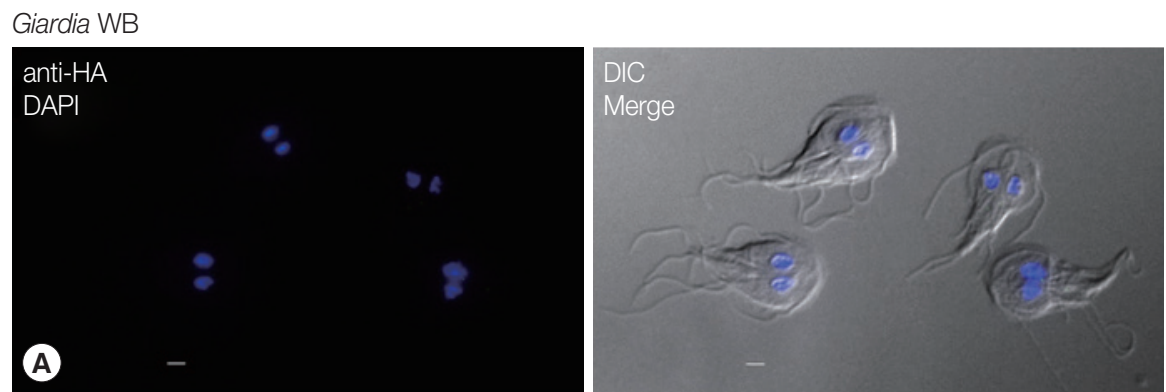

Giardia with HA tagged glmbp1
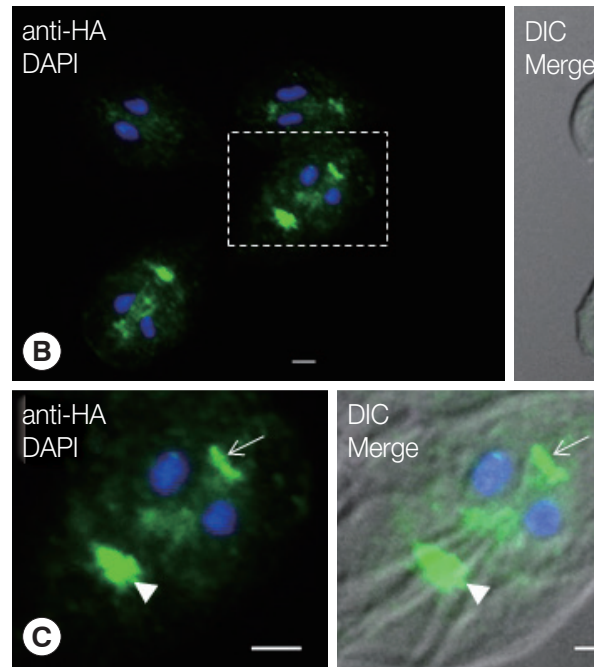

$\mathrm{DIC}$
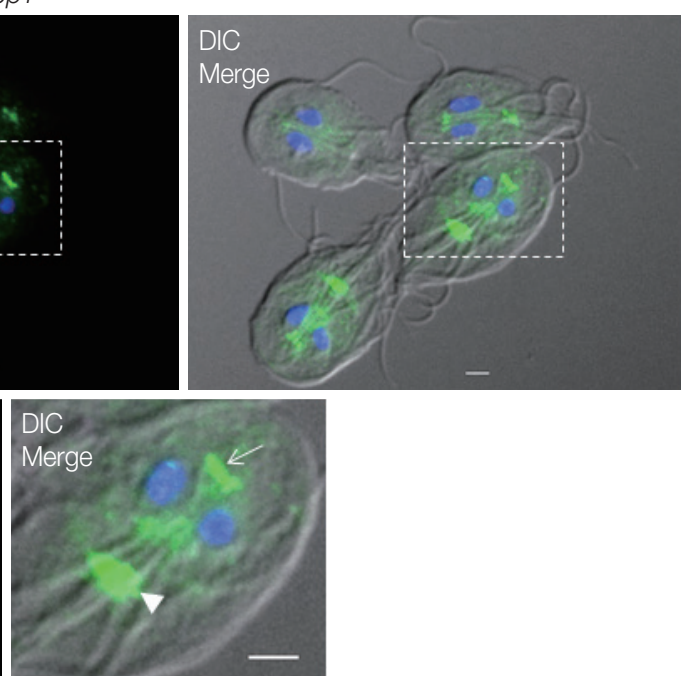

Fig. 5. Localization of GIMBP1 in G. lamblia trophozoites. G. lamblia cells expressing HA-tagged GIMBP1 were fixed with chilled 100\% methanol, and then permeabilized with PBS/0.5\% Triton X-100. The cells were reacted with mouse anti-HA antibodies (1:50 dilution) and then incubated with AlexaFluor 488-conugated anti-mouse lgG (1:200). The cells were mounted on slides with VECTASHIELD Antifade Mounting Medium with 4',6-diamidino-2-phenylindole, and then observed with an Axiovert 200 fluorescence microscope (Carl Ziess). Differential interference contrast (DIC) images showed cell morphology. (A) IFA on G. lamblia WB using anti-HA antibodies. The bars indicate $2 \mu \mathrm{m}$. (B, C) IFA on G. lamblia with integrated HA-tagged glmbp1 using anti-HA antibodies. The bars indicate $2 \mu \mathrm{m}$. Arrowheads indicate median bodies, while arrows show basal bodies.

$48 \mathrm{kDa}$, the expected size of GIMBP1, was apparent (Fig. 3B).

\section{Intracellular location of GIMBP1 in Giardia trophozoites}

In the next experiment, we examined whether GIMBP1 was localized in the cytoskeletal structures of G. lamblia. First, the glmbp1 gene tagged with HA epitopes was integrated by electroporation of linear glmbp1-HA DNA into the genomes of $G$. lamblia trophozoites (Fig. 4A, B) [15]. Integration of the chimeric glmbp1-HA gene into the Giardia chromosome was examined by PCR using glmbp1-specific primers and a primer specific for the vector plasmid, the pac reverse primer (Fig. 4C). A $1.6 \mathrm{~kb}$ glmbp1 PCR product was detected in both $G$. lamblia WB as well as in pGIMBP1HA.pac-transfected cells. A $2.5 \mathrm{~kb}$ PCR product, the expected length of glmbp 1 and the integrated plasmid, was amplified only from the genomic DNA of G. lamblia transfected with pGIMBP1HA, but not from that

\section{of G. lamblia WB.}

Giardia trophozoites with the integrated HA-tagged glmbp1 gene were then examined for the expression of a chimeric GIMBP1-HA by western blotting with anti-HA antibodies (Fig. 4D). An immunoreactive protein band of $48 \mathrm{kDa}$ was only present in the extracts of Giardia with the integrated HA-tagged glmbp1 gene (Fig. 4D). Giardia WB extracts did not show the immunoreactive protein. These results indicated that the HAtagged glmbp1 and pac genes were integrated into a G. lamblia chromosome, and that HA-tagged GlMBP1 was expressed in $G$. lamblia transfected with linear pGIMBP1HA.pac.

IFA was performed on G. lamblia WB and G. lamblia with the integrated glmbp1-HA gene using anti-HA antibodies (Fig. 5). G. lamblia WB trophozoites did not show any fluorescence in the IFA (Fig. 5A). In contrast, transgenic G. lamblia with HAtagged glmbp1 showed green fluorescence at axonemes, medi- 
an bodies, and basal bodies (Fig. 5B, C). This result indicated that GIMBP1 was located in cytoskeletal structures of G. lamblia trophozoites.

\section{DISCUSSION}

The cytoskeleton of G. lamblia is largely made of MTs, which includes a ventral disc, a median body, 8 basal bodies, and 4 pairs of flagella (anterior, posterior-lateral, ventral, and caudal). Each of the flagella is extended from an axoneme that is templated in a basal body. Basal bodies are conserved MT-organizing centers that nucleate structural apparatus such as flagella and cilia, and that function as spindle poles during cell division [16]. In G. lamblia, these structures were shown to be associated with MTs by electron immunocytochemistry [17] as well as IFA using anti-tubulin antibodies (Kim and Park, an unpublished result). Interestingly, $\gamma$-tubulin was found by IFA using monoclonal antibodies specific to $\gamma$-tubulin, only in the basal bodies of ventral and posterior-lateral flagella in the interphase Giardia cells. Further, this protein disappeared at early mitosis and reappeared in late-mitotic G. lamblia [18]. An extensive analysis of $G$. lamblia, based on in silico and proteomic methods, revealed 75 homologs of conserved basal body proteins. Thirteen of these homologs were found, by confocal microscopy, to co-localize with centrin in Giardia basal bodies [19]. Even though an association between GIMBP1 and basal bodies has not been reported, one of the MT-associated proteins was found to localize to cytoskeletal structures (axonemes, basal bodies, and median bodies) (Figs. 2, 5B).

In vitro interaction of GlMBP1 with MTs (Fig. 2) and its localization in the Giardia cytoskeleton indicates a role for GIMBP1 in cell cycle-related functions. However, the information available on GIMBP1 is too limited to allow a conclusion about its relationship with $G$. lamblia cell cycle. Monitoring the intracellular locations of GIMBP1 at various stages of G. lamblia will be essential to defining its involvement in cell cycle control in this organism. In addition, knockdown of glmbp1 expression will provide insight into the function of this protein in G. lamblia.

In summary, a novel binding protein was isolated while screening for MT-binding protein. Association of this protein with MTs was confirmed by in vitro MT-binding assays using rGIMBP1 and polymerized MTs. The intracellular location of this protein in G. lamblia was observed using transgenic $G$. lamblia in which an HA-tagged glmbp1 gene was integrated into a chromosome. IFAs using anti-HA antibodies revealed that GIMBP1 is located in cytoskeletal structures, median bodies, basal bodies, and axonemes. The physiological role of this GIMBP1-MT interaction should be defined in future investigations.

\section{ACKNOWLEDGMENT}

This research was supported by Basic Science Research Program through the National Research Foundation of Korea (NRF) funded by the Ministry of Education (2015R1D1A1A0 9057595).

\section{CONFLICT OF INTEREST}

We have no conflict of interest related to this work.

\section{REFERENCES}

1. Elmendorf HG, Dawson SC, McCaffery JM. The cytoskeleton of Giardia lamblia. Int J Parasitol 2003; 33: 3-28.

2. Desai A, Mitchison TJ. Microtubule polymerization dynamics. Annu Rev Cell Dev Biol 1997; 13: 83-117.

3. Akhmanova A, Steinmetz MO. Tracking the ends: a dynamic protein network controls the fate of microtubule tips. Nat Rev Mol Cell Biol 2008; 9: 309-322.

4. Dawson SC, Sagolia MS, Mancuso JJ, Woessner DJ, House SA, Friz-Laylin L, Cande WZ. Kinesin-13 regulates flagellar, interphase, mitotic microtubule dynamics in Giardia intestinalis. Eukaryot Cell 2007; 6: 2354-2364.

5. Kim J, Nagami S, Lee KH, Park SJ. Characterization of microtubule-binding and dimerization activity of Giardia lamblia endbinding 1 protein. PLoS One 2014; 9: e97850.

6. Kim J, Sim S, Kim J, Song K, Yong TS, Park SJ. Giardia lamblia EB1 is a functional homolog of yeast Bim1p that binds to microtubules. Parasitol Int 2008; 57: 465-471.

7. Holberton DV, Ward AP. Isolation of the cytoskeleton from Giardia. Tubulin and a low-molecular-weight protein associated with microribbon structures. J Cell Sci 1981; 47: 39-166.

8. Lourenço D, Andrade Ida S, Terra LL, Guimarães PR, Zingali RB, de Souza W. Proteomic analysis of the ventral disc of Giardia lamblia. BMC Res Notes 2012; 5: 41.

9. Hagen KD, Hirakawa MP, House SA, Schwartz CL, Pham JK, Cipriano MJ, De La Torre MJ, Sek AC, Du G, Forsythe BM, Dawson SC. Novel structural components of the ventral disc and lateral crest in Giardia intestinalis. PLoS Negl Trop Dis 2011; 5: e1442.

10. Woessner DJ, Dawson SC. The Giardia median body protein is a ventral disc protein that is critical for maintaining a domed disc conformation during attachment. Eukaryot Cell 2012; 11: 292301. 
11. Keister DB. Axenic culture of Giardia lamblia in TYI-S-33 medium supplemented with bile. Trans R Soc Trop Med Hyg 1983; 77: 487-488.

12. Harlow E, Lane D. Immunizations. In Antibodies: A Laboratory Manual, Immunization. New York, USA. Cold Spring Harbor Laboratory. 1988. pp 53-63.

13. Singer SM, Yee J, Nash TE. Episomal and integrated maintenance of foreign DNA in Giardia lamblia. Mol Biochem Parasitol 1998; 92: 59-69.

14. Kim J, Bae S, Sung M, Lee K, Park SJ. Comparative proteomic analysis of trophozoites versus cysts of Giardia lamblia. Parasitol Res 2009; 104: 475-479.

15. Gourguechon S, Cande WZ. Rapid tagging and integration of genes in Giardia intestinalis. Eukaryot Cell 2011; 10: 142-145.

16. Debec A, Sullivan W, Bettencourt-Diaz M. Centrioles: active players or passengers during mitosis? Cell Mol Life Sci 2010; 67: 21173-2194.

17. Crossley R, Marshall J, Cark JT, Holberton DV. Immunocytochemical differentiation of microtubules in the cytoskeleton of Giardia lamblia using monoclonal antibodies to $\gamma$-tubulin and polyclonal antibodies to associated low molecular weight proteins. J Cell Sci 1986; 80: 233-252.

18. Nohynkova E, Draber P, Reischig J, Kulda J. Localization of gamma-tubuin in interphase and mitotic cells of a unicellular eukaryote, Giardia intestinalis. Eur J Cell Biol 2000; 79: 438-445.

19. Lauwaet T, Smith AJ, Reiner DS, Romijn EP, Wong CCL, Davids BJ, Shah SA, Yates JR, Gillin FD. Mining the Giardia genome and proteome for conserved and unique basal body proteins. Int J Parasitol 2011; 41: 1079-1092. 